Acta Crystallographica Section D

Biological

Crystallography

ISSN 0907-4449

\title{
Protein microcrystals and the design of a micro- diffractometer: current experience and plans at EMBL and ESRF/ID13
}

There is a growing demand for the examination of protein microcrystals at third-generation synchrotron sources. After successful pilot experiments at EMBL/ESRF, which proved that protein microcrystals are often suitable for data collection, operation of the microfocus beamline ID13 was made more user-friendly and suitable for macromolecular crystallography experiments. Given the excellent quality of the beamline microfocusing optics, the key element for successful experiments becomes the handling and visualization of microcrystals. To address this, a microdiffractometer has been designed to allow maximum precision combined with ease of usage and is currently under construction.

Correspondence e-mail: perrakis@embl-grenoble.fr
C) 1999 International Union of Crystallography Printed in Denmark - all rights reserved

\section{Introduction}

Crystallographic structure solution for a wealth of proteins and macromolecular complexes is often hindered by the small size of the available crystals (Cusack et al., 1998). Moreover, despite diffracting X-rays to sufficient resolution in one or two reciprocal-space directions, crystals of particular shapes, such as thin plates and long but thin needles, may yield diffraction data of clearly inferior quality in the other direction(s) owing to increased background and insufficient irradiated volume. In some cases, when twinning or mosaicity are the problem, small crystals can be of superior quality to larger ones. Unfortunately, macromolecular microcrystals cannot be used in conventional sources or even at weaker synchrotron sources. A crystal of volume $V$ and unit-cell volume $V_{c}$ in a beam of intensity $I$ will yield diffraction peaks of an average intensity approximately proportional to $I\left(V / V_{c}\right)$. Thus, to obtain diffraction of equal strength from a $20 \mu \mathrm{m}$ and a more typical $200 \mu \mathrm{m}$ crystal, the former will require a $10^{3}$ increase in beam intensity, which should stay focused into that $20 \mu \mathrm{m}$ crystal. Furthermore, when a $200 \times 200 \times 20 \mu \mathrm{m}$ plate-shaped crystal is irradiated by a $200 \mu \mathrm{m}$ beam in the 'face-on' orientation, the crystal diffracts all the incoming X-rays. When the same crystal is hit 'edge-on' with the same beam, only one-tenth of the incoming X-rays will be diffracted by the crystal volume, resulting in diffraction peaks of lower intensity and increased background, since the same total crystal volume is irradiated with fewer X-rays. Microheterogeneity in the crystal (which is often observed for plate-shaped macromolecular crystals) will deteriorate data quality even further. Providing optics which can focus X-rays into very small volumes and thus match the smallest dimension of the crystal to the beam size is the solution expected to permit optimal use of such microcrystals.

However, successful data collection on such crystals will always require the surmounting of some severe obstacles. After a certain point, which remains to be clarified in practical terms (efforts have been made by Gonzalez \& Nave, 1994, and
Received 6 May 1999 Accepted 5 July 1999 
this issue has been theoretically considered by Henderson, 1995), too much X-ray energy is being deposited into a small volume. The protein structure and crystalline order are then destroyed very rapidly by primary radiation damage. Practical experience shows that cryocooling does not prevent severe radiation damage at $100 \mathrm{~K}$ if the $\mathrm{X}$-ray dose is as high as that obtained from a focused undulator source on a third-generation synchrotron. Owing to this damage, multiple crystals will generally be required for a full data collection, since use of ultra-short wavelengths or liquid-helium temperatures has not shown promising results (S. Ginel and G. Rosenbaum, personal communication; Perrakis et al., unpublished results; R. Ravelli \& S. Mac Sweeney, personal communication). Finally, one must consider that the fact that crystals do not grow any bigger often simply implies poor crystal quality, and not all microcrystals can be produced of sufficient quality for the collection of useful diffraction data.

\section{Protein microcrystallography at present}

Currently, macromolecular microcrystallography experiments at EMBL Grenoble/ESRF are being carried out on the ID13 microfocus beamline (Engström et al., 1995) and have produced some important biological structures (PebayPeyroula et al., 1997). Following this pioneering work, the setup was improved and made more user-friendly, with regard to both the beam characteristics and the sample environment. Our initial experiences with various projects which we thought could benefit from microfocusing are positive, and a brief overview of the possibilities which we believe routine microfocusing beam usage will offer are presented.

\subsection{Beamline ID13 characteristics}

The optical scheme used is based on an ellipsoidal mirror as the unique focusing element in combination with a $\mathrm{Si}(111)$ double monochromator. Given the present surface quality of mirrors, a focal-spot size of about $40 \mu \mathrm{m}$ (horizontal) $\times 20 \mu \mathrm{m}$ (vertical) and a divergence of about $2 \mathrm{mrad}$ (horizontal) $\times$ $0.2 \mathrm{mrad}$ (vertical) can be achieved (Riekel et al., 1997). Collimators of 10 or $30 \mu \mathrm{m}$ are placed in the focal spot, which define the final beam size and reduce the background. The optical system of ID13 is sufficiently stable to allow operation over several days with beam sizes down to a few micrometres; the beam drift is small and automatically corrected. The focal spots routinely achievable, 10 and $30 \mu \mathrm{m}$, are sufficient for macromolecular applications. Although the current limits of X-ray focusing are in the range 50-100 nm (Jark et al., 1996), monochromatic single-crystal diffractometry below the $10 \mu \mathrm{m}$ barrier will require much higher precision goniometers and, in particular, advances in sample observation and alignment.

\subsection{The sample environment}

Protein microcrystallography requires an order of magnitude higher precision (i.e. micrometres compared to tens of micrometers) in all related operations compared with normal operations. The principal problem is to be certain that the microcrystal is in the microbeam and on the rotation axis.

Visualization of tiny frozen crystals in loops is not always easy, and a crucial aspect of the optical visualization is correct illumination. In our experience, diffused light (using flexible fibre optics and reflecting the direct light either through a plastic screen or from a shiny surface) gives the best results. Two high-powered microscopes, one mounted vertically and the other mounted horizontally, facilitate visualization of microcrystals. This allows centring of the crystal without rotating the $\varphi$ axis, while the different qualities and directions of the two cameras offer increased flexibility in visualization. The first camera is bright, generally having low contrast, and is best suited for most crystals. The second camera is less bright and is most useful for visualizing thin needles without obtaining a 'lens' effects from the surrounding liquid. Some examples of current visualization capabilities are demonstrated in Fig. 1.

Since the recording of weak diffraction intensities is required, the background arising from air scattering must be minimized. Mounting of a very small beamstop close to the crystal position on the downstream side and an aperture on the upstream side very close to the crystal position help to eliminate air scatter. Both these components are motorized and can be moved towards or away from the crystal within a few seconds, allowing better access for sample mounting by the users.

Centring a thin glass needle predetermines the rotation centre. It is then efficiently aligned to the microbeam by using a bismuth germanate, $\mathrm{B}_{14} \mathrm{Ge}_{3} \mathrm{O}_{12}$, oxide (BGO) fluorescent crystal to visualize the beam position in both microscopes. The experimental table is moved so that the centre of rotation coincides with the beam.

Beam stability was increased by an automatic feedback system to maximize delivered intensity by adjusting the mirror and monochromator. A diode to monitor the delivered intensity is mounted on a pneumatic valve which can be operated remotely outside the experimental hutch.

The beamline is equipped with a MAR CCD $130 \mathrm{~cm}$ detector, which clearly demonstrated the value of a fast readout detector on such an intense beamline. Rapid read-out in many cases delivered useful data from a single crystal before radiation damage took place, although we cannot provide firm proof of the time-dependence of the radiation-damage process.

\subsection{Experience with microcrystals and why they need a microbeam}

Several experiments have been performed on the microfocus beamline ID13 with an undulator beam focused to $30 \mu \mathrm{m}$ at the sample position with an intensity of about $10^{12}$ photons s $^{-1}$ at various wavelengths between 0.78 and $1.0 \AA$. These experiments have established that there is ample flux density to obtain excellent high-resolution diffraction patterns in a few seconds even from very small crystals. 
Our experience to date has shown that different microcrystals show very different behaviour. Crystals of dimensions less than $50 \mu \mathrm{m}$ have been seen to diffract to resolutions of better than $2.0 \AA$ or not at all, to survive the

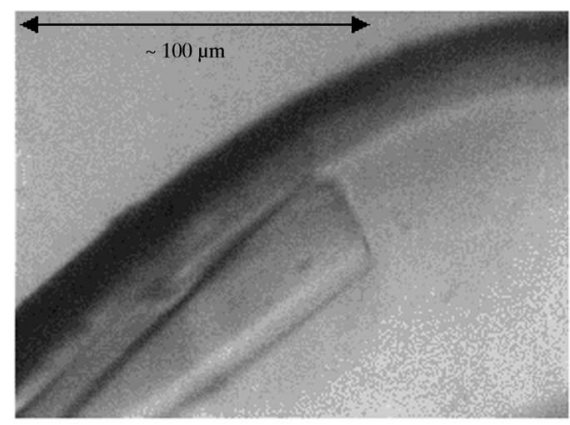

(a)

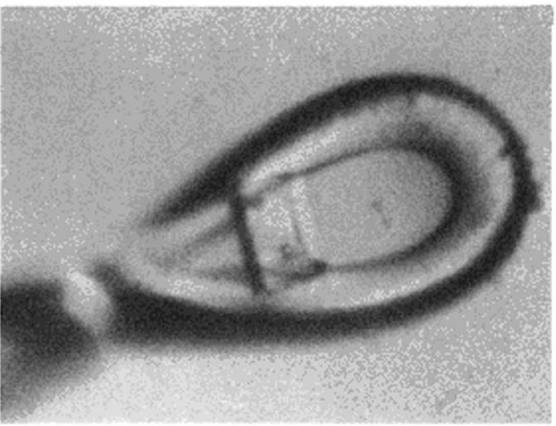

(c)

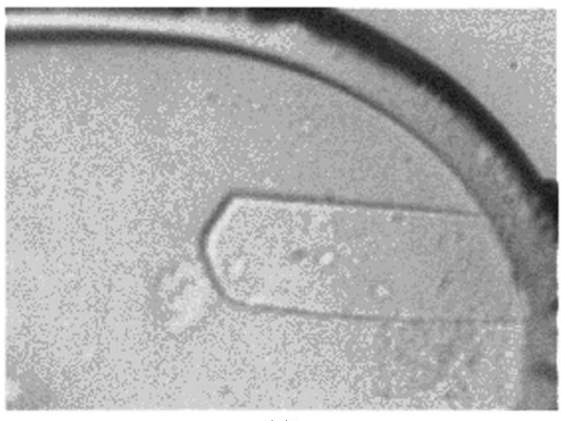

(e)

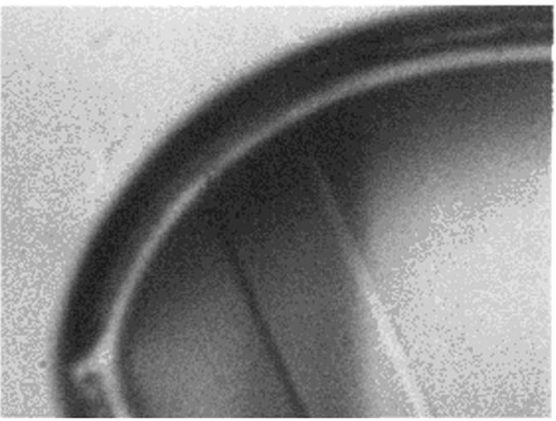

(g)

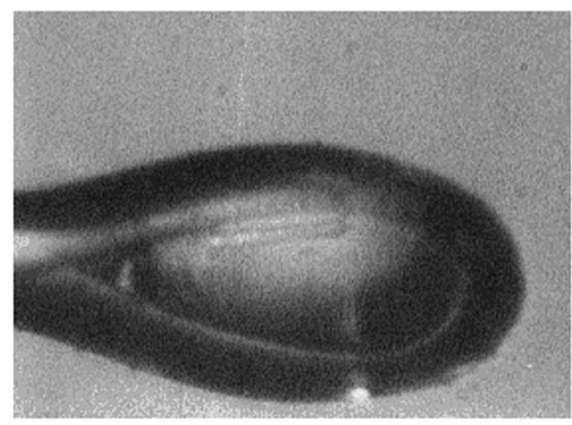

(b)

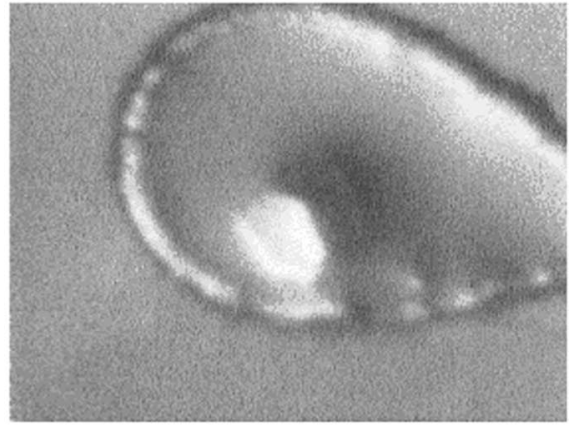

(d)

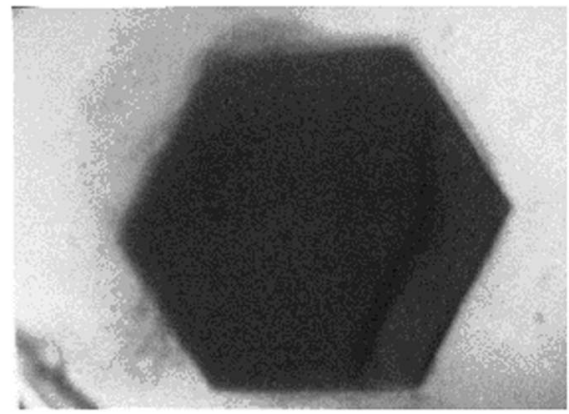

(f)

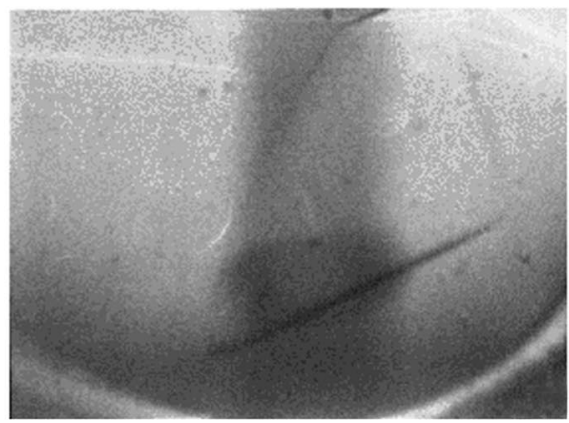

(h)
Figure 1

Visualization of different crystals with the current microfocus optics. In $(a)$, the $20 \times 10 \times 50 \mu \mathrm{m}$ crystal looks decently sized; in $(b)$, the thickness of the needle is clearly less than $5 \mu \mathrm{m}$; in $(c)$ and $(d)$, the largest dimension of these crystals does not exceed $50 \mu \mathrm{m} ;(e)$ and $(f)$ demonstrate the visualization of plates thinner than 2-3 $\mu \mathrm{m}$, while in $(g)$ and $(h)$ the path of the beam (the darker area) when shooting a crystal in one direction ( $g$, dark stripe) or two perpendicular directions ( $h$, dark stripe and darker spot) can be observed. The darker colour (blue in the colour camera) has often been observed in cryo-protectants containing polyethylene glycol. microbeam flux from only one exposure or to allow the collection of complete data sets, and to show variable degrees of mosaicity. In our experience, there is no safe way to predict the behaviour of such crystals, since home sources or even other synchrotron beamlines cannot provide the answers.

Two important applications of microcrystal diffraction are for needle-like and plate-like crystals. Diffraction data quality from such crystals can be superior owing to the better matching of the beam size to the sample in two orientations for needles or in one orientation for plates. Progressively translating such crystals in the microbeam, successively exposing $30 \times 30 \mu \mathrm{m}$ sections, has proven useful in avoiding radiation damage in a number of applications. Another potential application is to select microvolumes of larger crystals, which could be particularly important in the case of systematically twinned crystals or those with an apparent high mosaicity.

Several issues regarding protein microcrystal diffraction still need to be assessed in a more systematic manner: the tolerance of microcrystals to the intense beam as a function of their shape, volume, cryocooling conditions (e.g. loops with better heat conductivity, different cryo-protectants), cryo-cooling media (increased flows for nitrogen, helium cooling) and radiation wavelength. Further automation, for example the possibility of 'scanning' along the axis of thin needles or the surfaces and axes of thin plates, will allow better assessment of the benefits of such approaches. A clear assessment of the improvement both in diffraction quality and, most importantly, in data quality using the specific advantages of the microbeam in different crystal cases still needs to be performed.

\section{Design of a new microdiffractometer}

\subsection{Why?}

The sample environment for microcrystals has evolved over the last couple of years, building on our experience and that of invited users. High-power optics, correct illumination, use of fluorescent crystals to locate the beam and a carefully designed beamstop and aperture have been incorporated. However, it was clear that a new design was necessary, incorporating all 
these systems in a single robust mechanical platform and a more user-friendly environment. Approaches which have survived for more than half a century, such as crystal centring with a goniometer key, needed to be abandoned in order to perform microcrystallography in an efficient way. Highprecision computer-controlled mechanical components performing trivial operations exist and the main target was to integrate them into a proper environment. For these reasons, the EMBL and ESRF have jointly undertaken the development of a diffractometer specifically for microcrystal applications. The idea is to eliminate manual intervention after the initial input of the crystal (which in principle could also be performed by means of an automatic sample changer) by automating the alignment of crystal, beam and goniometer axis.

\subsection{Technical specifications for the microdiffractometer}

Each component of the microdiffractometer was designed with the main emphasis on precision and the user-friendliness of the environment. The design is shown in Fig. 2 and consists of a simple $\varphi$-rotation axis. Emphasis has been given to several technical innovations, which are discussed below.

3.2.1. The coaxial sample-visualization camera. A key element of the system is a high-powered microscope which looks along the beam direction to the sample position, thus avoiding parallax problems. A $10 \times$ microscope objective lens with a $33 \mathrm{~m}$ working distance is used to allow high magnification together with a large work space. The lens geometry has been redesigned to give a passage for the

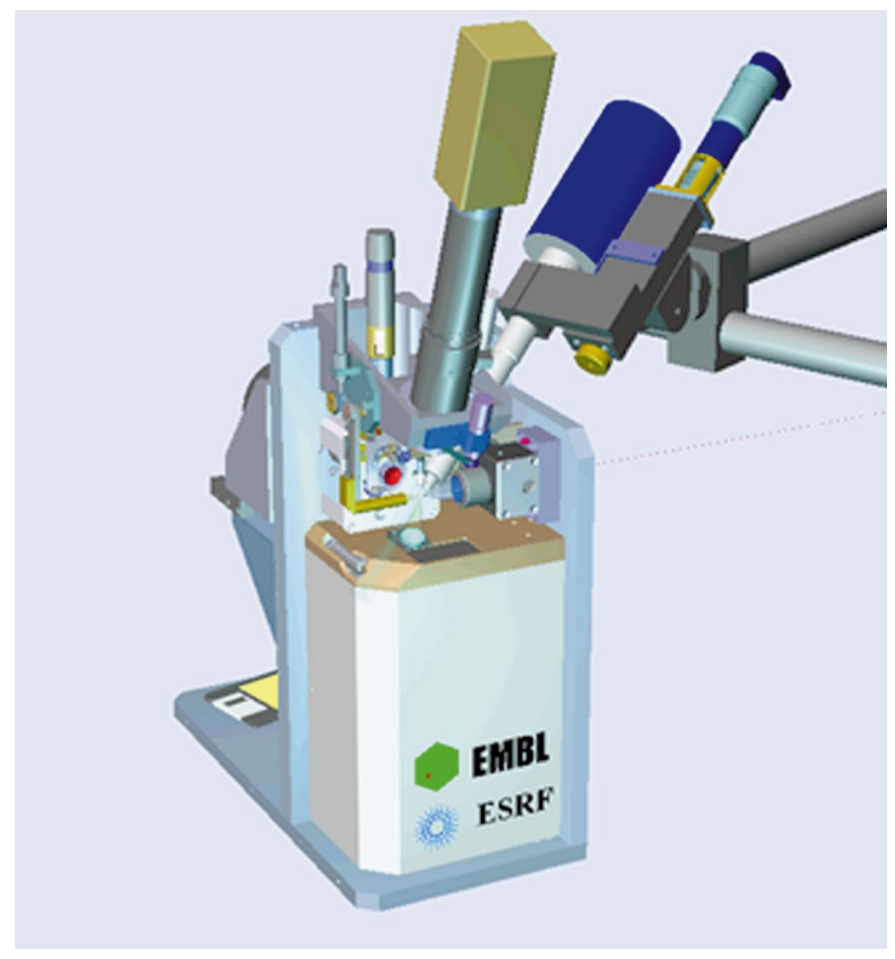

Figure 2

A global view of the microdiffractometer. All drawings are the rendered actual mechanical designs. beam by drilling holes in the first components and introducing a $45^{\circ}$ tilted mirror. The holes are coated with molybdenum to protect the lens from the direct beam. Thus, the first components are coaxial to the beam to allow coaxial visualization, while the remaining components (after the $45^{\circ}$ mirror) are at a $90^{\circ}$ angle, providing space for the placement of the other camera components. The lens is coupled with a $10 \times$ zoom and a CCD colour camera. This is directly mounted to a real-time video board on a PC. Having the image directly on the PC will allow future incorporation of automated methods for crystal alignment and image-enhancement algorithms to aid crystal visualization.

3.2.2. Auxiliary microscope. The auxiliary microscope with a $1.5 \times$ objective lens is mounted vertically and has a motorized zoom. This will facilitate sample location.

3.2.3. Sample centring. The idea of using a goniometer head is abandoned entirely. The sample holder is attached to a metal plate which is in contact with a magnetic base. Both the base and the plate are coated with a sliding carbon coating. Thus, the sample holder can be slid along the base, propelled by a motorized 'finger'. This procedure allows multiple degrees of freedom in centring (as opposed to the $\pm 90^{\circ}$ restrictions of the standard goniometer system) and minimizes mechanical 'backlash' which would occur if a 'screw-based' system was chosen, while offering a correction of up to $\pm 1.5 \mathrm{~mm}$ around the true rotation axis. All the above components are marked in Fig. 3.

3.2.4. Sample holder. The sample holder (marked in Fig. 3) can accommodate any kind of magnetic or mechanical base fitting the experiment, such as the 'Hampton' or 'Oxford' magnetic bases commonly used by protein crystallographers. Exchange can be performed very easily.

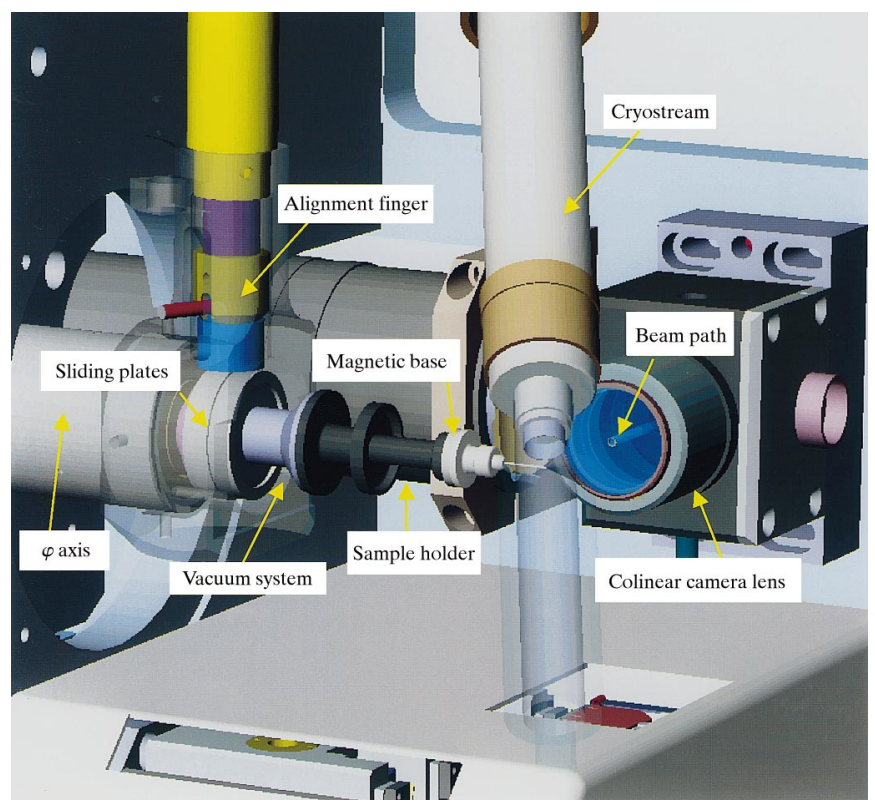

Figure 3

A close-up view of the sample environment. Important components are labelled. 
3.2.5. $\varphi$ axis. The rotation axis is based on a spindle axis coupled directly to a DC motor and controlled by a pulse encoder. The sphere of confusion is $\sim 1 \mu \mathrm{m}$ with a dynamic angular error of $\pm 2 \times 10^{-3 \circ}$. The rotation speed can vary from 0.02 to $1^{\circ} \mathrm{s}^{-1}$. The $\varphi$ axis is synchronized with the shutter though a hardware position comparator in the encoder.

3.2.6. Motorized arc. A motorized arc (Fig. 4) was designed to assist in sample mounting and sample recovery. The arc chariot together with the sample holder is first slid along the arc to a vertical position. The sample holder is then retracted to the horizontal position and fixed in place with a vacuum suction system. The chariot is retracted and the $\varphi$ axis is free for data collection.

3.2.7. Background-noise reduction. A small beamstop close to the crystal and a capillary aperture are used to minimize air scattering. They can be fitted on a magnetic base with an auto-centring system and will be easily exchangeable, in case they are damaged or the user desires different specifications. They are motorized in a vertical stage and can be completely retracted, so the user never really sees them in practice (Figs. $4 a$ and $4 b$ ). A security beamstop is downstream towards the detector to protect it from primary beam damage in case the primary beamstop is damaged or misaligned.

3.2.8. The scintillator. This is a small disc made from a $B G O$ crystal. It allows visualization of the location of the beam position with the same camera as the sample, thus permitting alignment of the sample and goniometer axis on the beam position. The expected precision is a few micrometers. It is mounted on a vertical stage and is fully retractable.

3.2.9. Graphical interface. A graphical interface, running under Windows NT, combining real-time sample visualization and assisting alignment, is under development.

The prototype microdiffractometer is under construction and should be ready for commissioning before the end of 1999 .

\subsection{How to handle a protein microcrystal in the year 2000}

The user will be able to bring his own mounting magnet for cryo-loops, which will first be adapted to the sample holder if necessary. As soon as the user enters the experimental hutch, the vertical stage with the diffractometer beamstop and aperture will be hidden. The user can then use all the space to mount the crystal in the sample holder, using vials with liquid nitrogen, ethane, propane or another system, transfer it with cryo-tongs or even freeze it on the cryostream (Fig. 4a). As soon as the sample is in place, the user can exit and interlock the hutch, without any further manual intervention being required.

From the diffractometer control PC, the user will then be guided through an alignment procedure. First, the sample should be located and the two extreme positions of the sample during a full-circle rotation will have to be marked on the screen by the user, using the mouse. The software will then perform the necessary translations to position the sample at the midpoint between the two marks corresponding to the rotation axis. In the future, one can contemplate algorithms for sample identification and complete automation of the procedure. After the sample is aligned to the rotation axis, the sample and the cryostream are moved by a few millimetres and the vertical stages containing the BGO scintillator and the safety beamstop are raised (Fig. 4b). The beam is then visualized on screen, using the same camera with which the crystal was located and aligned. The whole $\varphi$ axis is the translated so that the pre-identified centre of rotation is aligned with the beam. In this way, it is always verified that the crystal is in the centre of rotation and that this coincides with the beam just prior to exposure. The crystal and the cryostream are moved to the previous position and data collection can commence (Fig. 4c). The whole procedure should take less than a minute.

Besides speeding up and increasing accuracy in standard experimental techniques, this opens possibilities for new experimental strategies. Very small crystals on the same loop can be individually targeted with the beam and data can be collected for as long as the crystal survived without suffering significant radiation damage. The next crystal can then be targeted, hopefully in a new orientation, and a complete data set can be collected. Long needles can be translated along their axis, in order to scan for better

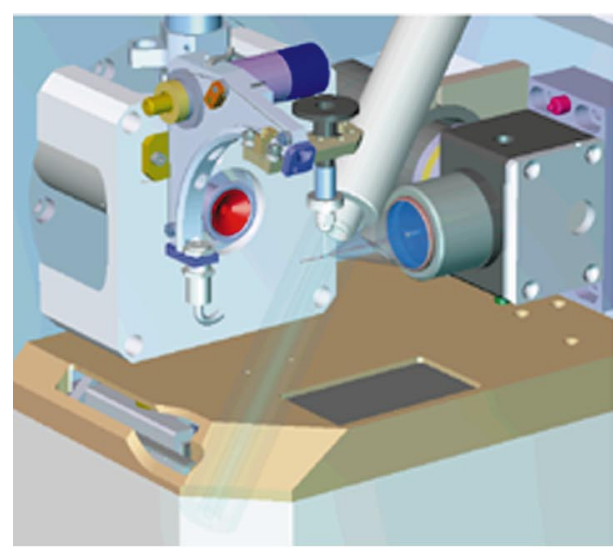

(a)

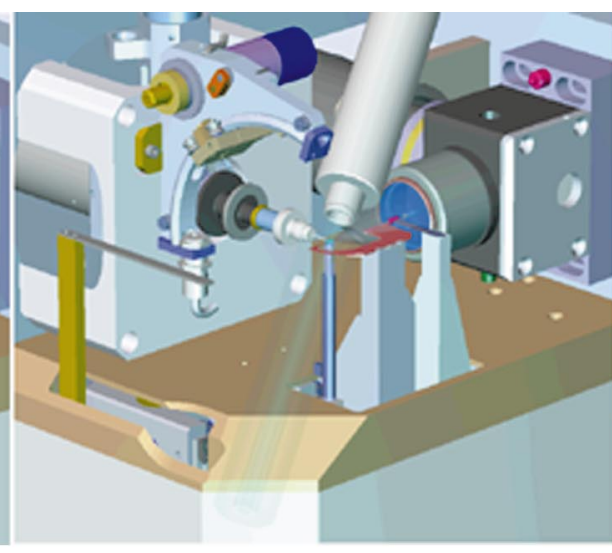

(b)

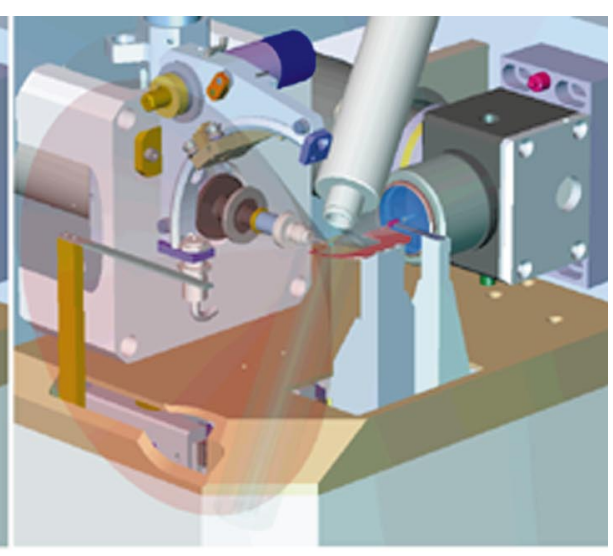

(c)

Figure 4

The sample-loading and centring sequence. See text for details. 
ordered regions or simply to reduce radiation damage. Very thin plates can also be translated for the above reasons and one can also optimize centring for edge-on diffraction by taking a few very short exposures, translating the crystal by a few micrometres in order to maximize the diffraction intensity.

The prototype microdiffractometer will be initially installed and tested in the microfocus beamline, where the benefits of its use are most obvious. The implementation of similar designs in other ESRF beamlines in the future is part of our plans and is expected to increase the ease, speed and quality of crystal mounting and alignment for all kinds of crystals. The microdiffractometer will be compatible with a crystal cryo-mounting robot which is under design by the Instrumentation group of the EMBL/ESRF JSBG (Andy Thompson \& Jean-Charles Castagna, personal communication).
We are very grateful to all the microfocus users for their contributions to testing the environment, their comments and suggestions.

\section{References}

Cusack, S., Belrhali, H., Bram, A., Burghammer, M., Perrakis, A. \& Riekel, C. (1998). Nature Struct. Biol. 5, Suppl. 634-637.

Engström, P., Fiedler, S. \& Riekel, C. (1995). Rev. Sci. Instrum. 66, 1348-1350.

Gonzalez, A. \& Nave, C. (1994). Acta Cryst. D50, 874-877.

Henderson, R. (1995). Quart. Rev. Biophys. 28, 171-193.

Jark, W., di Fonzo, S., Lagomarsino, S., Cedola, A., di Fabrizio, E., Bram, A. \& Riekel, C. (1996). J. Appl. Phys. 80, 4831-4836.

Pebay-Peyroula, E., Rummel, G., Rosenbusch, J. P. \& Landau, E. M. (1997). Science, 277, 1676-1681.

Riekel, C., Cedola, A., Heidelbach, F. \& Wagner, K. (1997). Macromolecules, 30, 1033-1037. 